\title{
ABDOMINAL COMPARTMENT SYNDROME (ACS): Presentation of a case series and proposal of UK Guidelines
}

\author{
Onyekwelu $\mathrm{O}^{2}$, Edwards $\mathrm{J}^{2}$, Falder $\mathrm{S}^{1}$, Ayub-Khan $\mathrm{H}^{2}$, Dunn $\mathrm{K}^{2}$. \\ Alder Hey Children's Hospital, Liverpool (1) and University Hospital of South Manchester (UHSM)(2)
}

Introduction

According to the World Society of the Abdominal Compartment Syndrome (WSACS) $)^{(1)}$ ACS may be defined as 'sustained intra-abdominal pressure (IAP) $>20 \mathrm{mmHg}$ concurrent with a new onset of organ dysfunction'(1). This state of organ failure frequently affects the cardiovascular, respiratory and renal systems (Fig. 1). ACS follows unrelieved progression of intra-abdominal hypertension (IAH). The condition is uniformly fatal if left untreated.

Ivy et al.(2) showed that fluid resuscitation volume of $0.25 \mathrm{~L} / \mathrm{kg}$ in the early post burn period resulted in IAP of $24.4 \mathrm{mmHg}$. The development of ACS in burn patients and association with high fluid resuscitation volumes is well known but this complication was the most frequent cause of death for significant burn patients in the UK in $2015 / 16$, suggesting that clinicians may be failing to consider this diagnosis or recognize the symptoms.

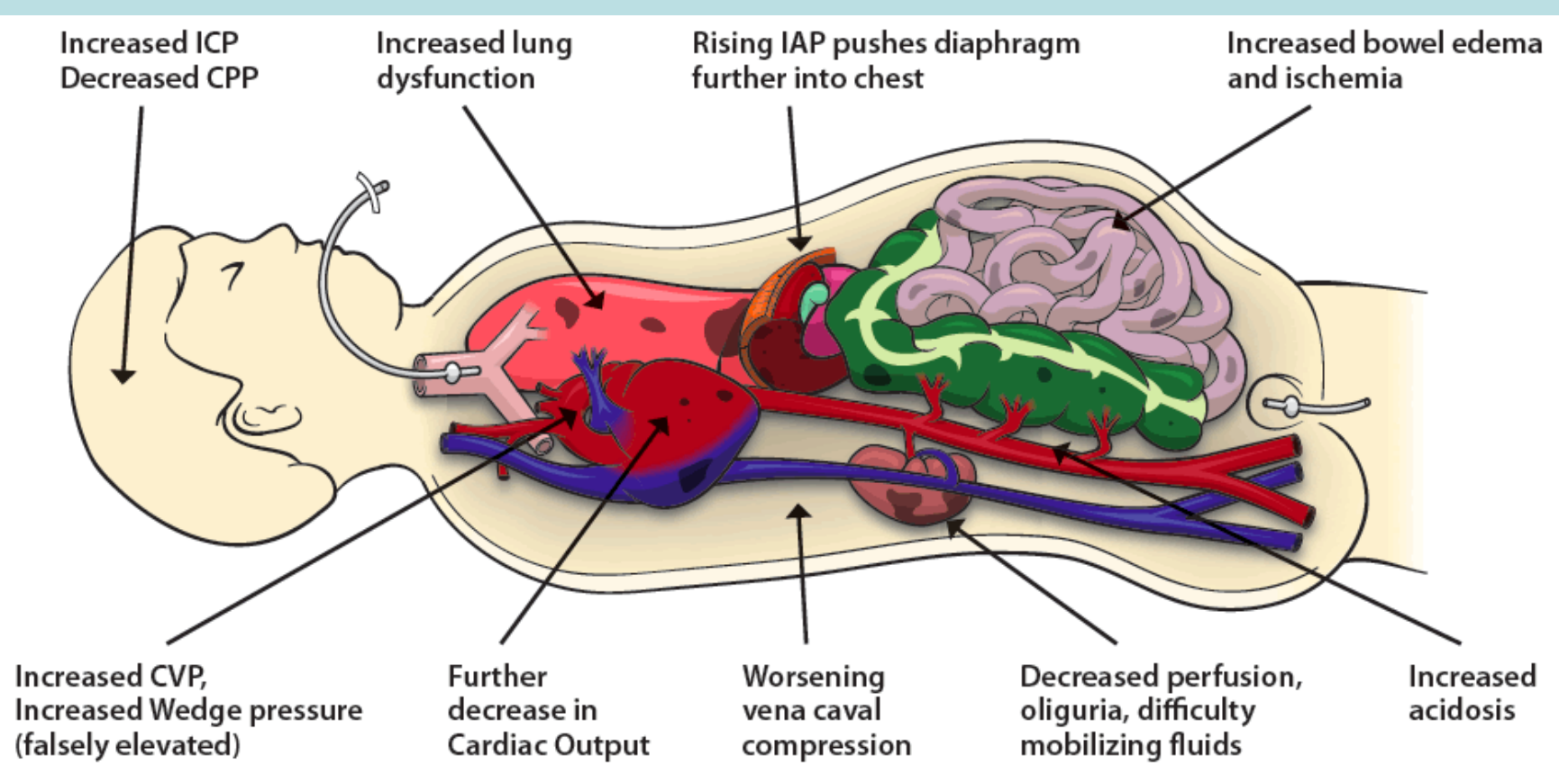

Fig 1.The pathophysiology of secondary ACS affects the entire body

\section{Discussion}

All 6 cases received more fluids than recommended by Parkland's formula in the first 24 hours and had high Ivy score. Two patients developed ACS within 4 days of injury. $5 \%$ human albumin solution in the early period (but after 8 hours) may reduce overall volumes required in the acute resuscitation phase. Our guidelines focus on strict fluid balance. Recording fluid balance using charts presenting total volume as $\mathrm{mls} / \mathrm{kg} / \% \mathrm{TBSA}$ burnt may raise awareness of fluid creep.

Four patients developed abdominal complications at days 28-46. Few cases had IAP measured until patients deteriorated. Signs and symptoms of ACS were not actively sought. WSACS management guidelines for ACS (Fig 2) dictate action dependent on IAP measurement and recommendations for frequency of IAP measurement.

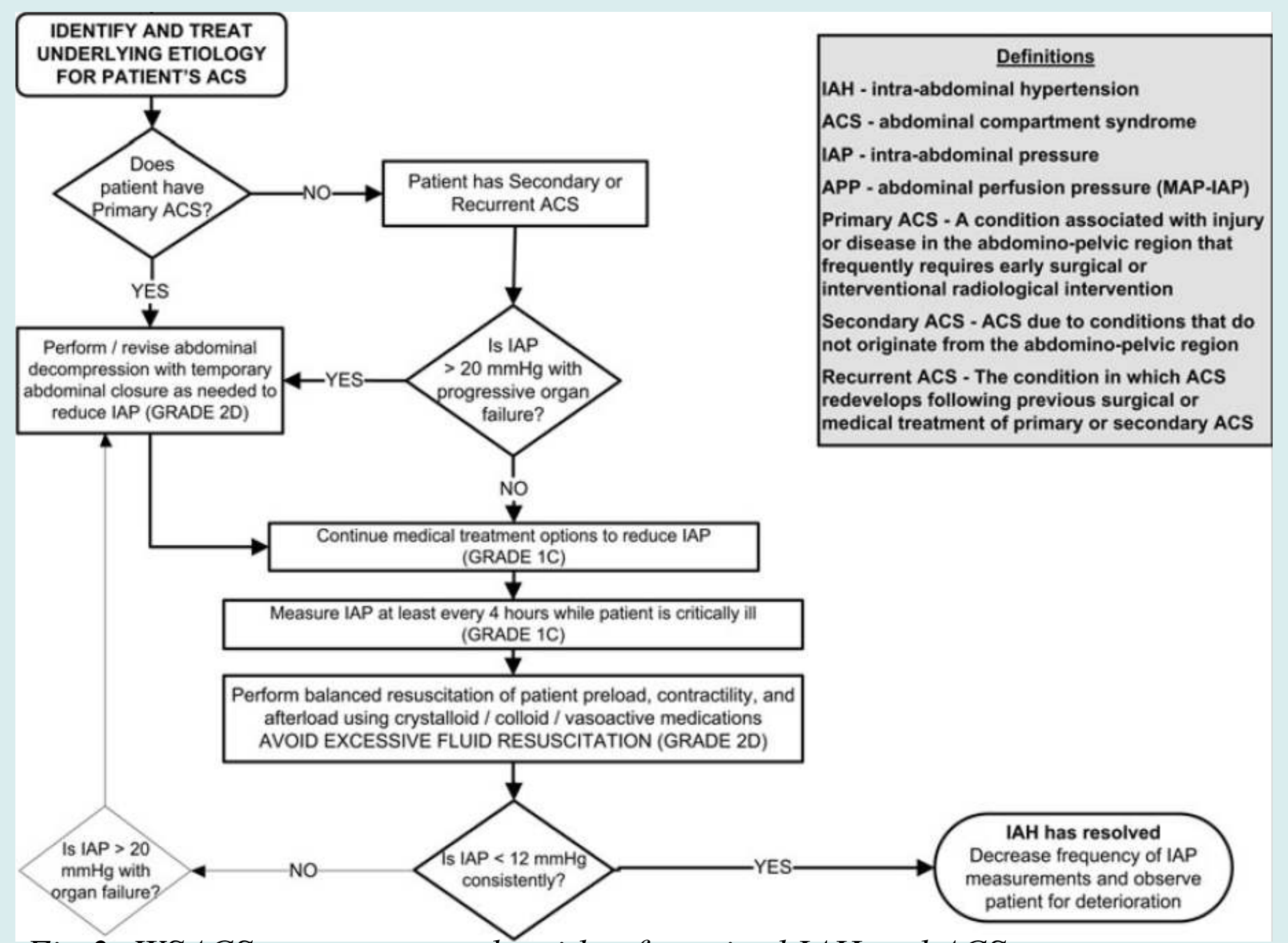

Fig 2: WSACS management algorithm for raised IAH and ACS $\underline{\text { Aim }}$

To audit patients with ACS in our burn units and propose guidelines to aid diagnosis and mitigate against development of ACS and bowel ischaemia.

Methods

Using the iBID database, we identified all patients who developed ACS, ischaemic bowel, or both ${ }^{(3)}$ in two burn units between Jan 2011 and Dec 2016. Fluid volumes in the first 24 hours, symptoms, diagnosis, management and outcome were recorded. Findings were analyzed by the clinical teams and suggested diagnostic and management guidelines agreed by consensus.

\section{$\underline{\text { Results }}$}

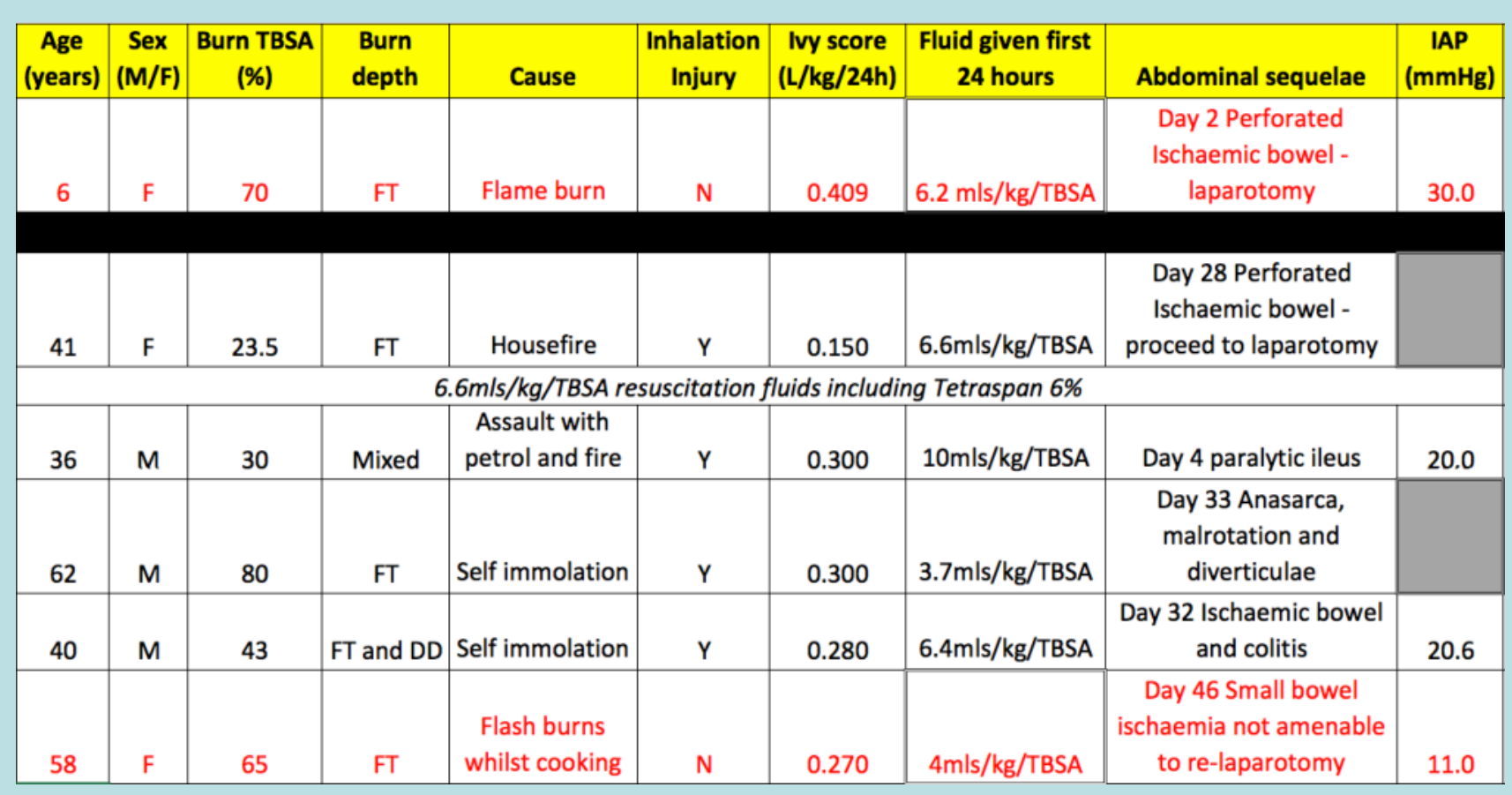

Strict fluid balance; measures to reduce total volumes; regular clinical examination in conscious patients; routine monitoring of IAP in ventilated patients may lead to earlier detection and treatment of IAH prior to development of ACS (Fig 3).

\section{Fig 3. Proposed awareness and management guidelines}

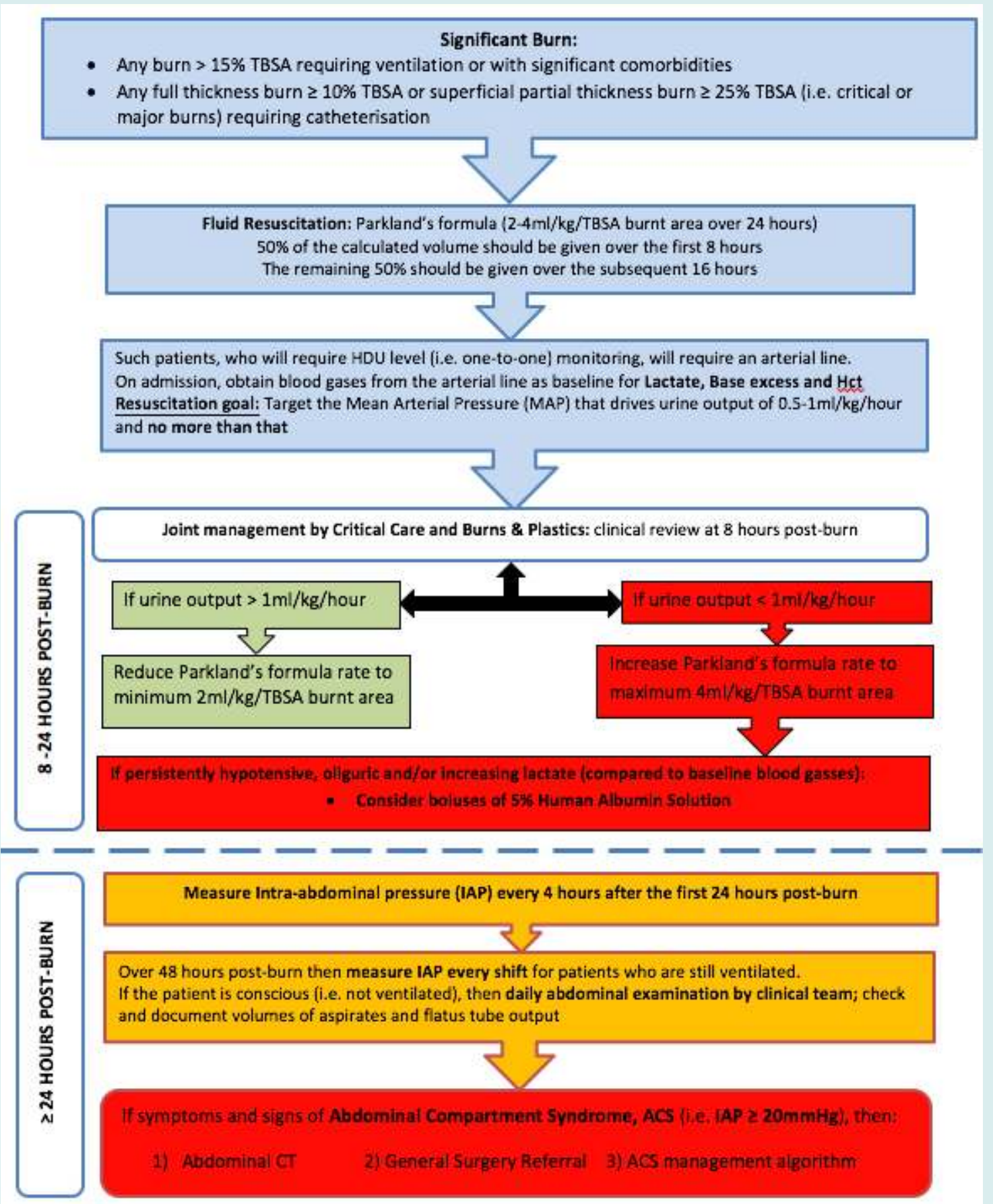
References:

1.Kirkpatrick AW et al., Intra-abdominal hypertension and the abdominal compartment syndrome: updated consensus definitions and clinical practice guidelines from the World Society of the Abdominal Compartment Syndrome. Intensive Care Med (2013) 39:1190-1206.

2.Ivy ME et al., Intra-abdominal hypertension and abdominal compartment syndrome in burn patients. $J$ Trauma 2000; 49(3): 387-91.

3.Wolfe SE et al., Abdominal Complications after Severe Burns. J Am Coll Surg Vol. 208, No. 5, May 2009. 